\title{
Manufacturing Defect Detection in Ceiling Fans using Image processing
}

\author{
Vaishali Malik, Pradeep Pant
}

\begin{abstract}
During manufacturing process of ceiling fans there may be possibility that any step of manufacturing process can be skipped or improper completion due to malfunctioning of the system. In manufacturing process of fans, the outer plate, stator, rotor, axle and other parts are manufactured and assembled. If the winding machine is not working properly and improper windings is done and no-one can acknowledge that winding machine is not working properly then the whole batch should be designed with defect and this will surely create negative impact on the production process of the industry. So this proposed work will overcome this problem. This paper guides to detect whether the windings are proper or not and detection is carried out by taking picture of armature-windings. If there is any problem in the windings then our system will generate an alert so that other armatures can be protected from failures. Manual results are not so accurate all the time but in manufacturing process we need high accuracy. For this skilled labor is required and for skilled labor we have to pay more, this automated system reduce the need of skilled labor. If fan manufacturing industries use this image processing system then this will be very helpful in increasing production of fans within the completion time with more accuracy.
\end{abstract} Rotor.

Keywords - Defect, Detection, Fan, Image, Manufacturing,

\section{INTRODUCTION}

Digital image processing is the process in which images are manipulated by means of computers. Image processing is growing rapidly among various technologies. Nowadays, digital image processing is widely used.

With the advancement of technology, digital images/digital systems are preferred over analog images/analog systems. Today, smart phone users are increasing day by day and everyone captures their moments in phones in the form of photos, videos and animations. Editing in photos and videos, applying filters to images can be done with the help of digital image processing techniques. So we can say that concept and application of the digital imaging is growing at rapid rate. To extract features from images, digital image processing is used. This is automatically done by computers with or without human involvement. Image processing is widely used in: pattern recognition, medical field, face detection, video processing, color, brightness and contrast management, fingerprint identification e.t.c. It is widely used in almost every field. Nowadays biometric attendance is mandatory in almost

Revised Manuscript Received on July 18, 2019.

Vaishali Malik,. Student, M. Tech., Computer Science \& Engineering from Meerut Institute of Engineering \& Technology (MIET), Meerut, Uttar Pradesh, India.

Pradeep Pant, Professor, Computer Science \& Engineering from Meerut Institute of Engineering \& Technology (MIET), Meerut, Uttar Pradesh, India. every organization which is the part of digital image processing.

Many industries use image processing to check whether the final product contain all elements or not, whether the shape of end product is correct or not, whether there is label on the packet or not, e.t.c. Maintaining quality of a product is the most important task for any industry.

In the past manual inspection has played a significant role for inspection of quality and verification of product. In modern industrial production lines, working speed should be high and minimum tolerances are required. Also, manual inspection is considered as limiting factor over modern automated systems. Traditional modes for defect detection are slow and may cause errors. So the introduction of artificial vision-based inspection [1] can be used as the solution to these types of problems. Many industrial sectors widely use applications of these systems.

The manual method for inspection of defects requires skilled labor, which is slow and may vary from observer to observer. Automatic packing and sorting lines already exist from many years. Automatic inspection for defected rotor has not been possible. Again human give their judgments on the basis of their knowledge and on the basis of what they expect from the product. Even most of the naive observers cannot identify the structure but still they agree that there is defect. Of course, such monitoring can give inaccurate results, may vary from observer to observer and expensive also. Due to all these reasons the need of automatic system for classification and detection of defect cannot be denied.

\section{OBJECTIVE}

Our research proposes an effective and efficient technique for defect detection with higher rates for image defect detection within minimum time.

This paper will meet the following objectives:

- To provide accurate results with precision.

- To detect defect in the windings of rotor.

- To detect defect in very short time.

\section{DEFECT DETECTION (EXISTING METHOD)}

In previous years, some methods have been proposed for detection of defects by using image. But these methods have some drawbacks which are discussed below:

H. Elbehiery et al. [7] presented some techniques to detect the defects. Their method is divided into two parts. For the first part, the input is the captured images of rotor (as per existing method). Existing method showed histogram

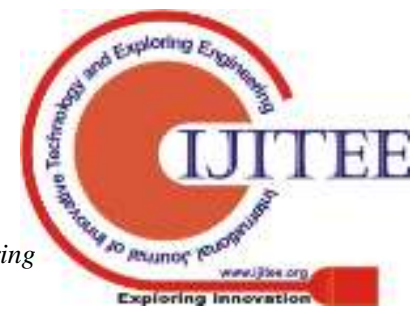


equalized image as output. After which the output of the first part is used as the input for the second part. In the second part of their algorithm, to identify different kinds of defects they have used different operations of image processing. In manufacturing process, automated system is very much necessary but their system is not automated. On every test image they have applied their second part, for the identification of various kinds of defects which makes their proposed method operation redundant. Also, this proposed method consumes more time.

\section{DEFECT DETECTION AND CLASSIFICATION (PROPOSED APPROACH)}

This section proposes a new method for detection of defect and its classification. This method has higher rate for defect detection than existing method and the total time for computation can be reduced. For detection of different kinds of defects in rotor a series of operations is adopted and to classify them some proposed algorithms are used. The proposed approach for defect detection and classification will be performed by using following steps:

Step 1: Perform preprocessing on image.

Step 2: Apply the proposed process for detection of defect.

Step 3: Classify the defect by using proposed algorithms.

In our proposed method, some preprocessing operations are performed on the image such as noise reduction, image enhancement, and e.t.c. For this proposed method, Sobel edge detection method [3] [4] is used on the resultant image to detect edge. There are many of edge detectors which are used to detect edges of the image. Here we have used first derivative edge detector (Sobel), due to its simpler calculation and it detects edges very fast. On the other hand, if second derivative edge detector operator such as Laplacian or Gaussian operator is used then finding the orientation of edge becomes difficult due to the use of Laplacian filter

In Fig.1 standard rotor image (original/standard/color image) is given, which is used to train the system and the system will perform comparison on the basis of this correct image. The system will compare this standard image with the input image and if there is difference in the threshold value then that image is said to be defected.

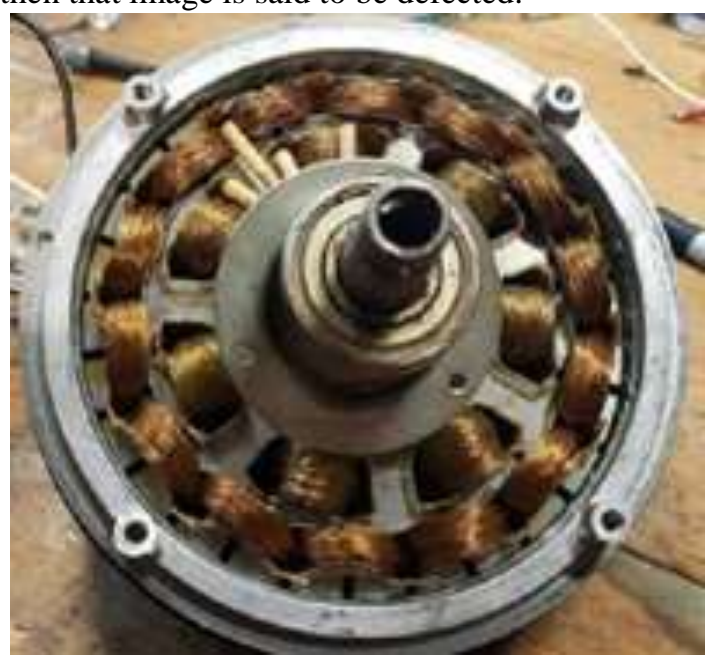

\section{TESTING RESULT}

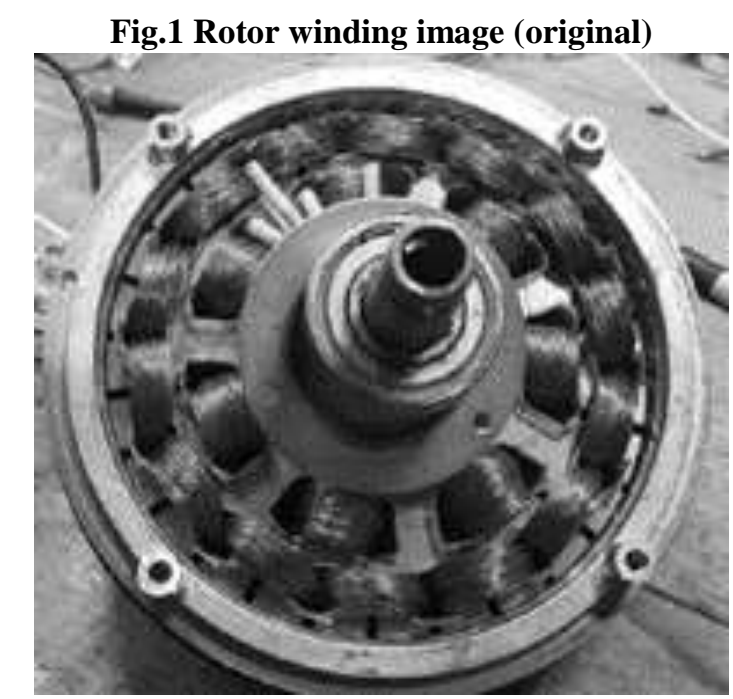

Fig.2 Rotor winding gray image (original)

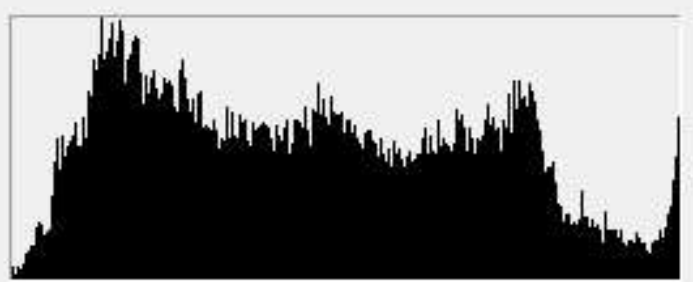

Fig.3 Rotor winding gray image histogram graph

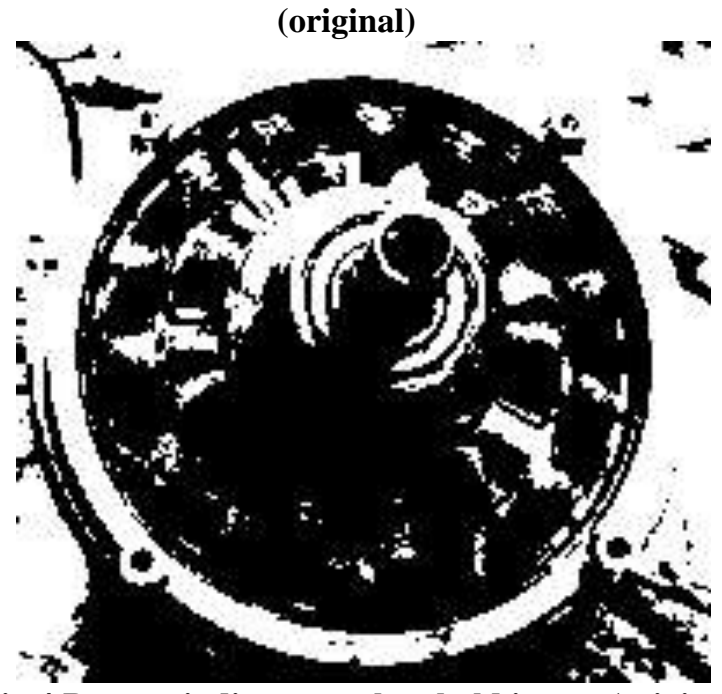

Fig.4 Rotor winding gray threshold image (original)

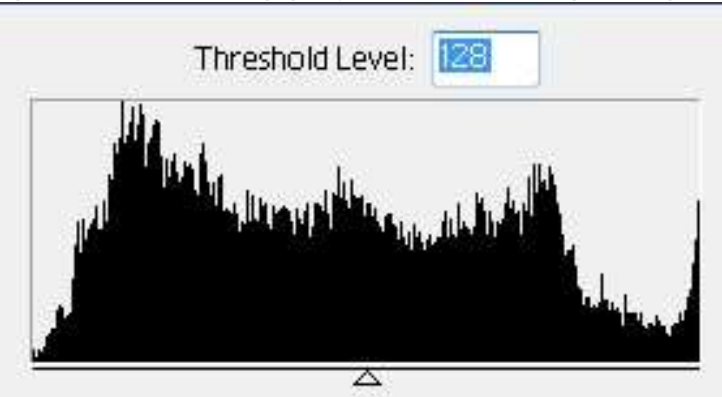

Fig.5 Rotor winding gray threshold image graph (original)

Fig .2 Shows the gray image of rotor winding. Fig.3 shows the histogram for that image then the threshold image 
of rotor windings are formed which is shown in Fig.4. Fig.5shows the histogram of the threshold image (threshold of Fig.4). Then the same procedure is done for testing. Fig.6 to Fig.10 shows the conversion of test image from colored image to gray image then its histogram is formed then threshold image is formed and then its histogram is obtained. Then threshold value is compared.

Test 1

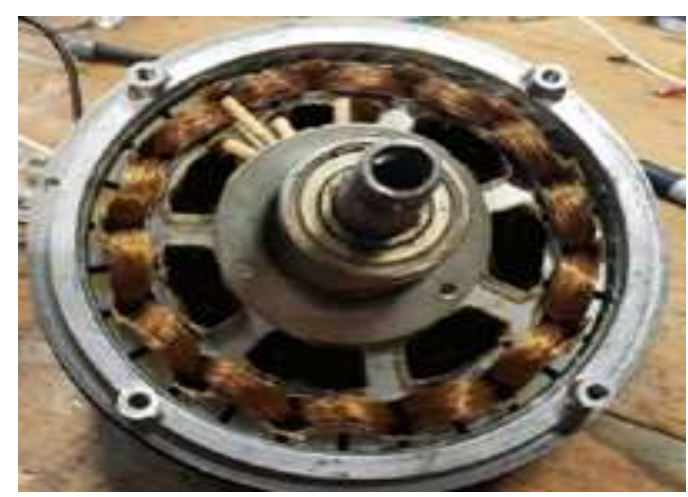

Fig.6 Defect 1 rotor winding image

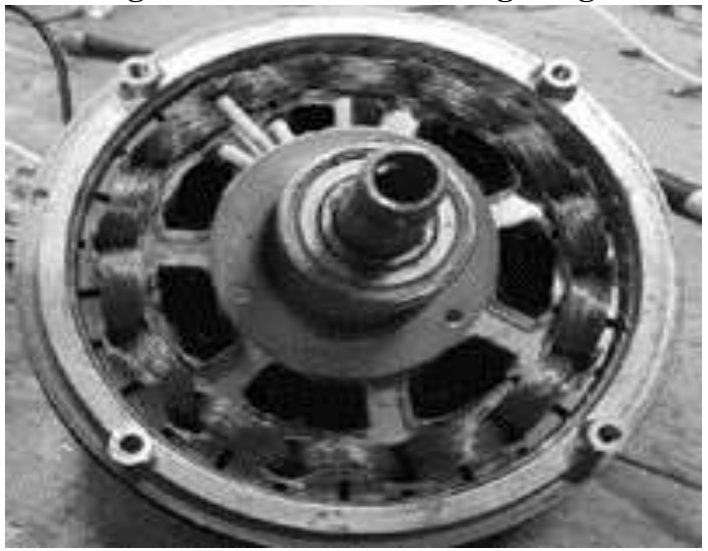

Fig.7 Defect 1 rotor winding gray image

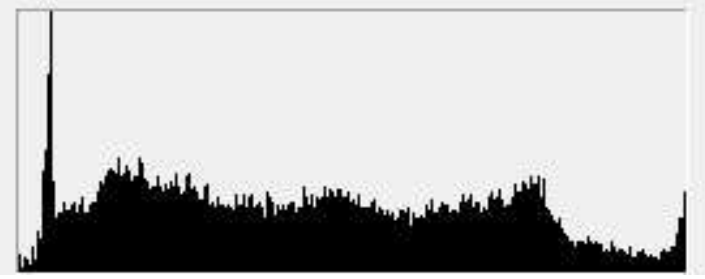

Fig.8 Defect 1 rotor winding gray image histogram

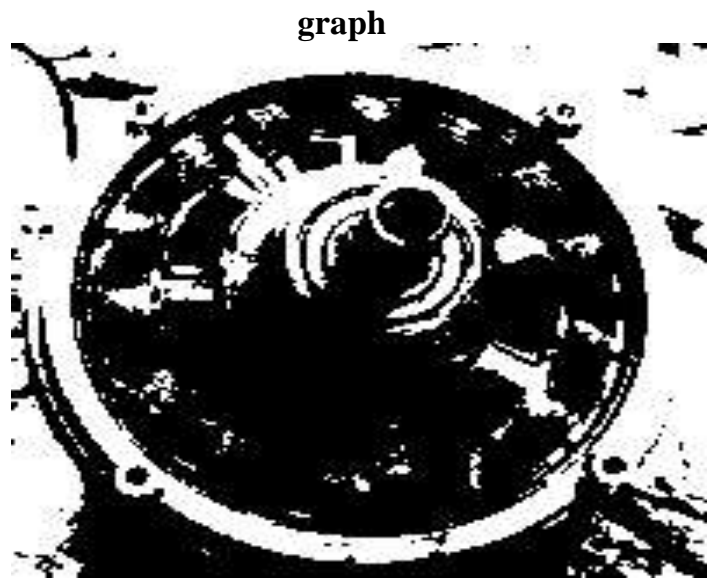

Fig.9 Defect 1 rotor winding gray threshold image

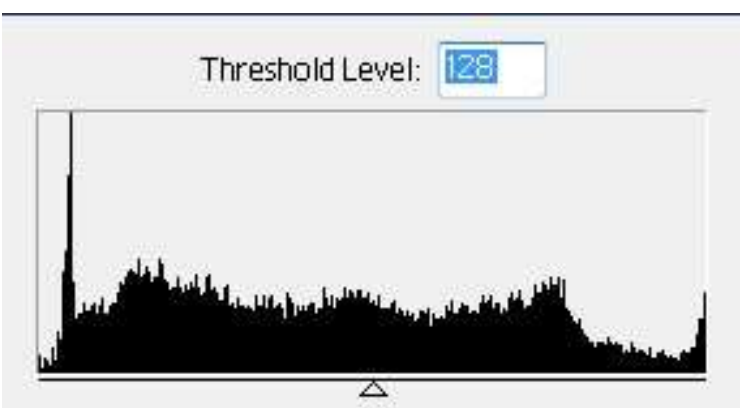

Fig.10 Defect 1 rotor winding gray threshold image graph

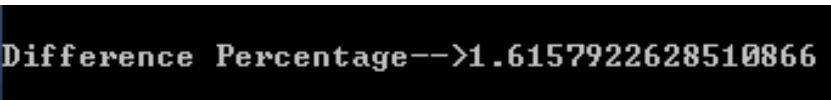

According to our proposed work, if the difference percentage is 0.00 then we can say that there is no defect. As shown in the above test 1 the difference percentage is 1.61579226285108 which indicates that there is defect.

\section{CONCLUSION}

Manufacturers can experience many significant benefits from automatic defect detection systems when used in-line. Such systems reduce number of defected products and improve quality of manufacturing industry, which leads to cost savings and raise the position of the company among other companies in this competitive environment. Most of the defect detection systems which are commercially available use dedicated circuits. With the constant improvements in performance of image sensors, decreased cost of processing electronic systems and increased power has increased the possibility of making defect detection system which is cost effective. This movement appears to be continued.

Specific edge detection technique is used for the detection of object from the image (considering external environment effect). For preprocessing step, contrast stretching is required which is fulfilled by equalizing the histogram. In real world environment, even if the method of object detection give better results but the time consumption is high then this not acceptable. In order to take less time for object detection various techniques such as smoothing, noise reduction, morphology operations can be used. Thus we have concluded that there is no such algorithm for finding all defects at a time.

\section{FUTURE WORK}

In future, to find all kinds of defects one generalized algorithm can be prepared.

In our future work our main focus is on:

1) Improvement in the efficiency of defect detection techniques by using different computational resources.

2) Reduction in the complexity of threshold and 
segmentation algorithms.

3) Working on multiple defects like scratches and many more by using single technique.

\section{REFERENCES}

1. E.N. Malamas, E.G.M. Petrakis, M. Zervakis, L. Petit, J.D. Legat, A survey on industrial vision systems, applications and tools, Image and Vision Computing 21, 2003, 171-188.

2. M.A.Coulthard, "Image Processing for Automatic Surface Defect Detection," Surface Inspection Ltd, UK, pp. 192-196.

3. Rafael C. Gonzalez and Richard E. Woods, third edition, Pearson Education International. "Digital Image Processing".

4. VineetSaini, RajnishGarg, IOSR Journal of Electronics and Communication Engineering, Volume 1, May-June 2012, ISSN: 2278-2834. "A Comparative Analysis on Edge Detection Techniques Used in Image Processing

5. R. C. Gonzalez, R. E. Woods, "Digital Image Processing", PearsonEducation (Singapore), Pte. Ltd., Indian Branch, 482 F.I.E. Patparganj,2005-2006.

6. Puyin Liu, Hongxing Li, "Fuzzy Neural Network Theory andApplication". World Scientific, 2004.

7. H. Elbehiery, A. Hefnawy, and M. Elewa, "Surface Defects DetectionUsing Image Processing and MorphologicalTechniques", Proceedings of World Academy of Science, Engineeringand Technology, vol 5, pp 158-160, April 2005, ISSN 1307-6884.

8. Se Ho Choi, Jong Pil Yun, BoyeulSeo, Young Su Park, Sang Woo Kim,"Real-Time Defects Detection Algorithm for High-Speed Steel Bar inCoil", Proceedings of World Academy of Science, Engineering andTechnology, Volume 21, January 2007, ISSN 1307-6884.

9. Mohamed Roushdi, "Comparative Study of Edge Detection AlgorithmsApplying on the Grayscale Noisy Image Using Morphological Filter",GVIP Journal, Volume 6, Issue 4, December, 2006.

10. Kumar, Tarun, and Karun Verma. "A Theory Based on Conversion of RGB image to Gray image." International Journal of Computer Applications 7.2 (2010): 7-10.

11. Min, Yongzhi, et al. "Real time detection system for rail surface defects based on machine vision." EURASIP Journal on Image and Video Processing 2018.1 (2018):3.

12. Nakano, Kanako, et al. "Detection of object in digital image." U.S. Patent Application No. 10/027,878.

\section{AUTHORS PROFILE}

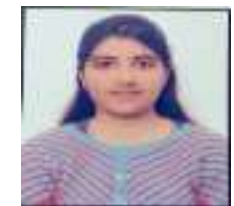

Vaishali Malik, pursuing M. tech in Computer Science \& Engineering from Meerut Institute of Engineering \& Technology (MIET), Meerut, Uttar Pradesh, India. Studied B. tech in Computer Science \& engineering from Dr. A. P. J. Kalam University, Lucknow, Uttar Pradesh, India. One survey paper on "Manufacturing Defect Detection in Ceiling Fans by using Image Processing" has been presented in 1st International Conference on Future Computing and Communication Technology which is published in International Journal of Applied Engineering Research.

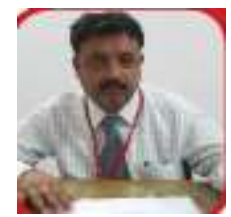

Prof. Pradeep Pant, Head of Department (H.O.D), Department of Computer Science \& Engineering, in Meerut Institute of Engineering \& Technology (MIET), Meerut, Uttar Pradesh, India Educational qualification: Ph. D in CSE from CMJ University, $\mathrm{M}$. tech in Computer engineering from Shobhit University, B. tech in CSE from Kumaon Engineering College and have 17 years of teaching experience. Many papers have been published in 7 journals and some are presented and published in International Conferences. Attended more than 20 FDP/ Workshops/ Seminars. 